JAIST Repository

https://dspace.jaist.ac.jp/

\begin{tabular}{|c|c|}
\hline Title & $\begin{array}{l}\text { An agent-based appr oach to i dent i f i cat } i \text { on of } \\
\text { pr edi ct i on model s }\end{array}$ \\
\hline Author(s) & Na, Ti ej u; Nakamor i , Yoshi t er u \\
\hline Citation & $\begin{array}{l}\text { I nt er nat i onal Journal of Uncert ai nt y, Fuzzi ness } \\
\text { and Know edge- Based Syst ens, 11( 4): 495-514 }\end{array}$ \\
\hline Issue Date & $2003-08$ \\
\hline Type & Journal Article \\
\hline Text version & aut hor \\
\hline URL & ht t p: //hdl . handl e. net /10119/5026 \\
\hline Rights & $\begin{array}{l}\text { El ect roni c ver si on of an art i cle publ i shed as } \\
\text { I nt er nat i onal Journal of Uncert ai nt y, Fuzzi ness } \\
\text { and Know edge- Based Syst ens, 11(4), 2003, 495- } \\
\text { 514. Dol : 10. 1142/S021848850300220X. Copyri ght } \\
\text { Wbr I d Sci ent i f i c Publ i shi ng Company, } \\
\text { ht t p: //dx. doi . or g/10. 1142/S021848850300220X }\end{array}$ \\
\hline Description & \\
\hline
\end{tabular}

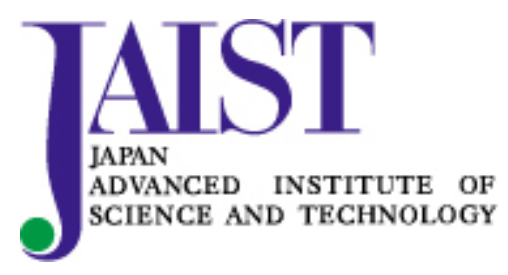


An Agent-Based Approach to Identification Prediction Models, the International Journal of Uncertainty, Fuzziness and Knowledge-Based Systems, World Scientific, vol 11.4, pp. 495-514, August 2003. (Tieju Ma with Yoshiteru Nakamori) 


\title{
AN AGENT-BASED APPROACH TO IDENTIFICATION OF PREDICTION MODELS
}

\author{
TIEJU MA and YOSHITERU NAKAMORI \\ School of Knowledge Science, Japan Advanced Institute of Science and Technology \\ 1-1 Asahidai, Tatsunokuchi, Ishikawa 923-1292, Japan
}

\begin{abstract}
This paper presents an agent-based approach to identification of prediction models in two-dimensional data spaces. A number of agents are sent to the two-dimensional data space that people want to investigate. At the micro-level, every agent tries to build a local linear model by competing with others, and then at the macro-level all surviving agents build the global model by cooperating with each other. And a genetic algorithm is introduced for improving the global model built by the agents. Two examples that apply this approach are given. The advantages of this approach are it does not need people to give a certain formula in advance; and most of time, it can give more precise prediction models than those given by traditional methods.
\end{abstract}

Keywords: agent, local linear model, global model, data space.

\section{Introduction}

During the 1980s and 1990s, many disciplines converged into an interdisciplinary research field, i.e. research on complex adaptive systems. A belief underlying this complex adaptive systems research is that macro-level complexity of the system may spontaneously be derived from the interactions at the micro-level and that this fundamental mechanism should be common among different systems in different fields. As a consequence, agent-based modeling has been applied to many fields in the last decade. Such works can be seen in Gerara Ballot and Erol Taymaz (1999) ${ }^{1}$, Miller John H. (1998) ${ }^{2}$, Jim Doran (1994) $)^{3}$, Ostrom Thomas M. $(1988)^{4}$, Beuu Roy $(1998)^{5}$, Derek W. Bunn and Fernando S. Oliveira $(2001)^{6}$, and so on.

Prediction can be viewed as the construction and use of a model to assess the class of an unlabeled sample, or to assess the value or value ranges of an attribute that a given sample is likely to have ${ }^{7}$. Prediction of discrete or nominal values can be dealt with by classification techniques, such as Classification by Decision Tree 
Induction, Bayesian Classification, Classification by Backpropagation, Classification Based on Concepts from Association Rule Mining, and so on. And for the prediction of continuous values, it is always modeled by statistical techniques of regression. We intend to apply agent technology to identify prediction models in a bottom-up way. As a first step of this plan, the approach presented in this paper deals with the prediction of continuous values with two dimensions.

Modeling by statistical techniques of regression is something like a top-down way. People first observe the distribution of all the data, form a whole structure of the data space in their minds, then select a certain function to fit the data by experience and specific knowledge. For example, for the data distribution in Fig. 1_a, people will select linear model $y=a x+b$; for the data distribution in Fig. 1_b, people may select exponential function $y=a^{x}+b$; and for the data distribution in Fig. 1_c, people may select sine or cosine function $y=a \sin x+b$ or $y=a \cos x+b$. But how about the data distribution in Fig. 2 or more complex distributions? It is very difficult or impossible to find a function to fit the whole data distribution by using statistical techniques of regression in a top-down way. Certainly we can divide all the data in Fig. 2 into several parts, and for every part we find a function to fit it. But when we deal with high-dimensional data, for example 6-dimensional data, how can we know where we should separate the data. This paper puts forward the idea that we can find the whole structure of data space by agent technology in a bottom-up way, and the approach presented in this paper is elicited by this basic idea.

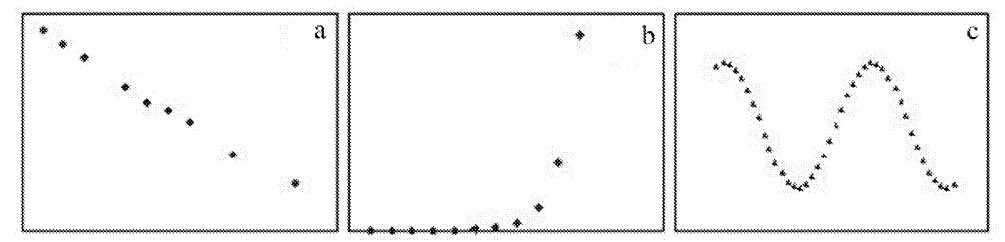

Fig. 1. Cases which are easy for modeling.

A simple description of the approach presented in this paper is as follows. A number of agents are sent to the two-dimensional data space that we want to investigate. At the micro-level, individual agents use linear regressions to build local linear models in their territories; at the same time, they try to expand their territories by finding new data objects in their visions. Correlation coefficients are used as rules to decide whether agents succeed in expanding their territory or not. During the process of expanding their territories, there is survival competition among agents. Then at the macro level, the local linear models built by those surviving agents are integrated into a global model by introducing membership functions and applying a genetic algorithm.

In our agent-based approach, we will introduce three design parameters which 

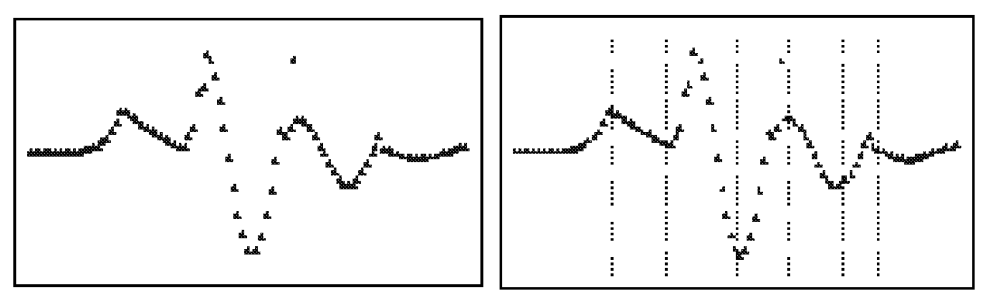

Fig. 2. A case which are difficult for modeling.

are $p_{1}, p_{2}$ and $p_{3}$. Considering the argument that "modeling is an art" 8 , Y. Nakamori point out that, without using educated and informed intuition, it is difficult to obtain a convincing model that can be used in an actual situation ${ }^{9}$. According with this argument, it is necessary to join logic and intuition together and avoid combinatorial explosion for setting the three parameters. The second numerical example in section 5 shows human's informed intuition can play an important role during the process of modeling.

\section{Standardizing Data Set}

Before investigating a data set, it is necessary to standardize it to simplify the later work. Suppose that the raw data set is $O=\left\{\left(x_{1}^{\prime}, y_{1}^{\prime}\right),\left(x_{2}^{\prime}, y_{2}^{\prime}\right), \cdots\left(x_{N}^{\prime}, y_{N}^{\prime}\right)\right\}$. We call $o_{i}\left(x_{i}^{\prime}, y_{i}^{\prime}\right) \in O$ a data object of data set $O$. It can be standardized in both $X$-dimension and $Y$-dimension with Eq. (1) and Eq. (2)

$$
\begin{aligned}
x_{i} & =\frac{\left(x_{i}^{\prime}-x_{\min }^{\prime}\right) \times \text { SpaceXSize }}{x_{\max }^{\prime}-x_{\min }^{\prime}}, \\
y_{i} & =\frac{\left(y_{i}^{\prime}-y_{\min }^{\prime}\right) \times \text { SpaceYSize }}{y_{\max }^{\prime}-y_{\min }^{\prime}} .
\end{aligned}
$$

Here $x_{i}^{\prime}$ indicates the value of $X$-dimension of object $o_{i}, x_{i}$ indicates the standardized $x_{i}^{\prime}, x_{\max }^{\prime}$ indicates the maximum value of $O$ in $X$-dimension, $x_{\min }^{\prime}$ indicates the minimum value of $O$ in $X$-dimension, SpaceXSize indicates the size of $X$-dimension of the space in which we will put all data objects; $y_{i}^{\prime}$ indicates the value of $Y$-dimension of object $o_{i}, y_{i}$ indicates the standardized $y_{i}^{\prime}, y_{\max }^{\prime}$ indicates the maximum value of $O$ in $Y$-dimension, $y_{\min }^{\prime}$ indicates the minimum value of $O$ in $Y$-dimension, and SpaceY Size indicates the size of $Y$-dimension of the space. The setting of SpaceXSize and SpaceYSize depends on the accuracy requirements of the result. If the accuracy requirements are high, they should be set big, otherwise they can be set a little small. In the rest of this paper, the setting of SpaceXSize and SpaceY Size also follows this rule: they are big enough to meet the accuracy requirements when all the values are standardized into integers. As an approach it is not necessary to follow this rule; the rule is used just for simplifying the work 
of programming. In the rest of this paper we also set SpaceXSize = SpaceYSize, which is also for simplifying the work of programming. After standardized the data set, we can build a data space and put all the data objects into it. As shown in Fig. 3 , points indicate the data objects.

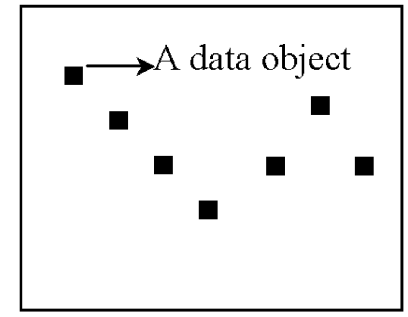

Fig. 3. Data space.

\section{Agents and Building Local Linear Model}

\subsection{Initializing Agents}

From a programming perspective, agent technology is a further development of the object-oriented technology. There are many definitions of agent. In this paper, an agent is simply defined as a programmed unit.

If totally there are $N$ data objects, then after building the data space, $N$ agents are sent into it, and each agent mounts on a data object. Each agent is initialized like the following:

Level: level $=1$,

Vision: $p_{1}$,

Strength: $s=0$.

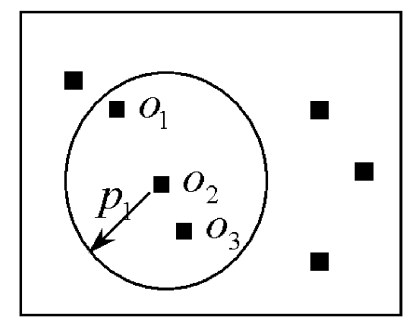

Fig. 4. Vision of an agent.

In our approach, agents will evolve through competition. Their initial level is 1 ; 
after competing with each other, some agents will evolve into high levels and some agents will die. Every agent has a vision, which means its ability to observe the environment. As shown in Fig. 4, in its field of vision (indicated by the circle), the agent mounting on data object $o_{2}$ can see another two data objects, $o_{1}$ and $o_{3}$. The $p_{1}$ can be seen as a design parameter, and the setting of it need human's informed intuition. An agent's strength indicates its power of building a global structure of the data space by cooperating with other agents. For an agent of level 1, its strength is 0 because it does not participate in the construction of the global structure that is done by higher level agents.

Besides the above properties, the agents in our approach also have built-in knowledge and instinct. Every agent knows the regression method, and it expands its territory by instinct.

\subsection{Building Local Linear Model}

After initialization, every agent tries to build a local linear model according to the following steps:

- Step 1: The agent counts the number of objects in its vision. If the number is smaller than 3, this agent dies; otherwise it goes to step 2.

- Step 2: The agent does linear regression analysis of all the data objects in its vision. If the correlation coefficient $r \geq p_{2}$, it goes to step 4; otherwise it go to step 3. Here $p_{2}$ is another design parameter.

- Step 3: The agent decreases its vision by 1 , that means $p_{1}^{t+1}=p_{1}^{t}-1$ (here $t$ means time). If $p_{1}^{t+1}=0$ it dies; otherwise it goes to step 1 .

- Step 4: The agent builds a local linear model and evolves into an agent with level 2 .

This progress also can be shown as in Fig. 5 .

\subsection{Expanding Territory}

Those agents that evolve into level-2 agents try to expand their territories. Here an agent's territory means the subspace that includes all the data objects used by the agent to build its local linear model. As shown in Fig. 6, if agent A builds a local linear model based on data objects $o_{1}, o_{2}, o_{3}, o_{4}$ and $o_{5}$, then its territory is the area which includes these five data objects. Although an agent's territory is shown as an ellipse in Fig. 6, it has no special shape. In fact it is a set of the data objects that are used by the agent to build its local linear model. Another thing worthy of attention is that a data object can be in several agents' territories. As shown in Fig. 6, the data objects $o_{4}$ and $o_{5}$ belong to both agent A's territory and agent B's territory. Also an agent can move in its territory. For example, the agent A in Fig. 6 can move from the position of data object $o_{1}$ to the positions of data object $o_{2}$, $o_{3}, o_{4}$ and $o_{5}$. 


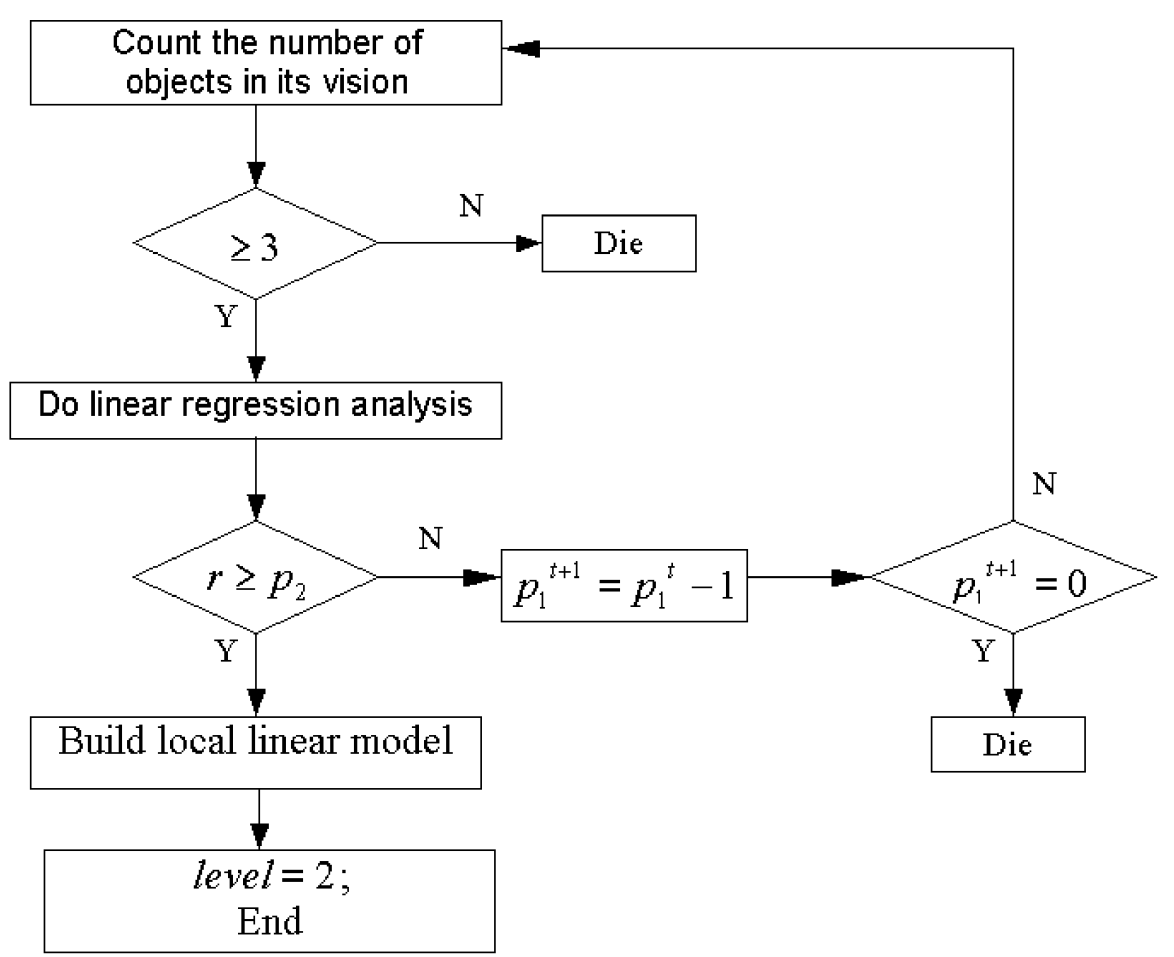

Fig. 5. The process of building local linear models.

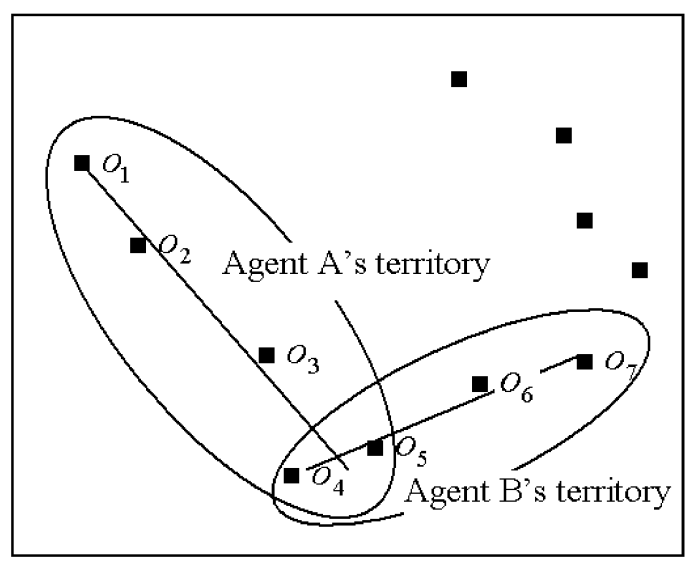

Fig. 6. Agents' territory. 
An agent expands its territory according to the following steps:

- Step 1: The agent finds all frontier data objects, and memorizes them in a list fo. The agent also memorizes its current territory as $T_{0}$.

- Step 2: The agent moves to the location of a frontier data object in its territory.

- Step 3: The agent looks for new data objects in its vision. If it finds new data objects, it goes to step 4; otherwise it goes to step 5 .

- Step 4: The agent does linear regression analysis with the data objects in $T_{0}$ and these new data objects. If the correlation coefficient $r \geq p_{3}$, the agent succeeds in expanding its territory, that is to say these new data objects are included in this agent's territory; otherwise it fails to expand its territory.

- Step 5: If there are another frontier data objects in list fo that the agent has not visited, it moves to one of them and goes to step 3. If the agent has visited all the data objects in the list $f_{o}$, it memorizes its current territory as $T_{1}$ and then goes to step 6.

- Step 6: If $T_{1}=T_{0}$, the agent stops expanding its territory and evolves into level 3 ; otherwise it goes to step 1.

This progress can be shown as in Fig. 7 .

An agent finds the frontier objects of its territory in the following way:

If the number of all data objects in its territory is $M$ and the distance between two data objects $o_{i}\left(x_{i}, y_{i}\right)$ and $o_{j}\left(x_{j}, y_{j}\right)$ is $d_{i j}=\sqrt{\left(x_{i}-x_{j}\right)^{2}+\left(y_{i}-y_{j}\right)^{2}}$, then the agent gets the distance matrix $D$ :

$$
D=\left[\begin{array}{cccc}
d_{11} & d_{12} & \cdots & d_{1 M} \\
d_{21} & d_{22} & \cdots & d_{2 M} \\
\vdots & \vdots & \vdots & \vdots \\
d_{M 1} & d_{M 2} & \cdots & d_{M M}
\end{array}\right]
$$

The agent ransacks $D$ and finds the biggest distance values. The data objects that form these biggest values are marked as frontier data objects by the agent. For example if $d_{23}$ is one of the biggest distance values, then data objects $o_{2}$ and $o_{3}$ are marked as frontier objects.

Along with the progress of agents' expanding their territories, there is competition among agents for survival in the data space. After an agent has expanded its territory (at the end of step 5), it checks whether there are other agents whose territories are covered by its territory. If there are, it eats those agents, i.e. those agents are removed from the data space. As shown in Fig. 8, agent A eats agent B. Those agents that expand their territories more efficiently and more effectively will have more chance to survive. In this sense, expanding its territory can be seen as a manifestation of an agents' instinct for survival. 


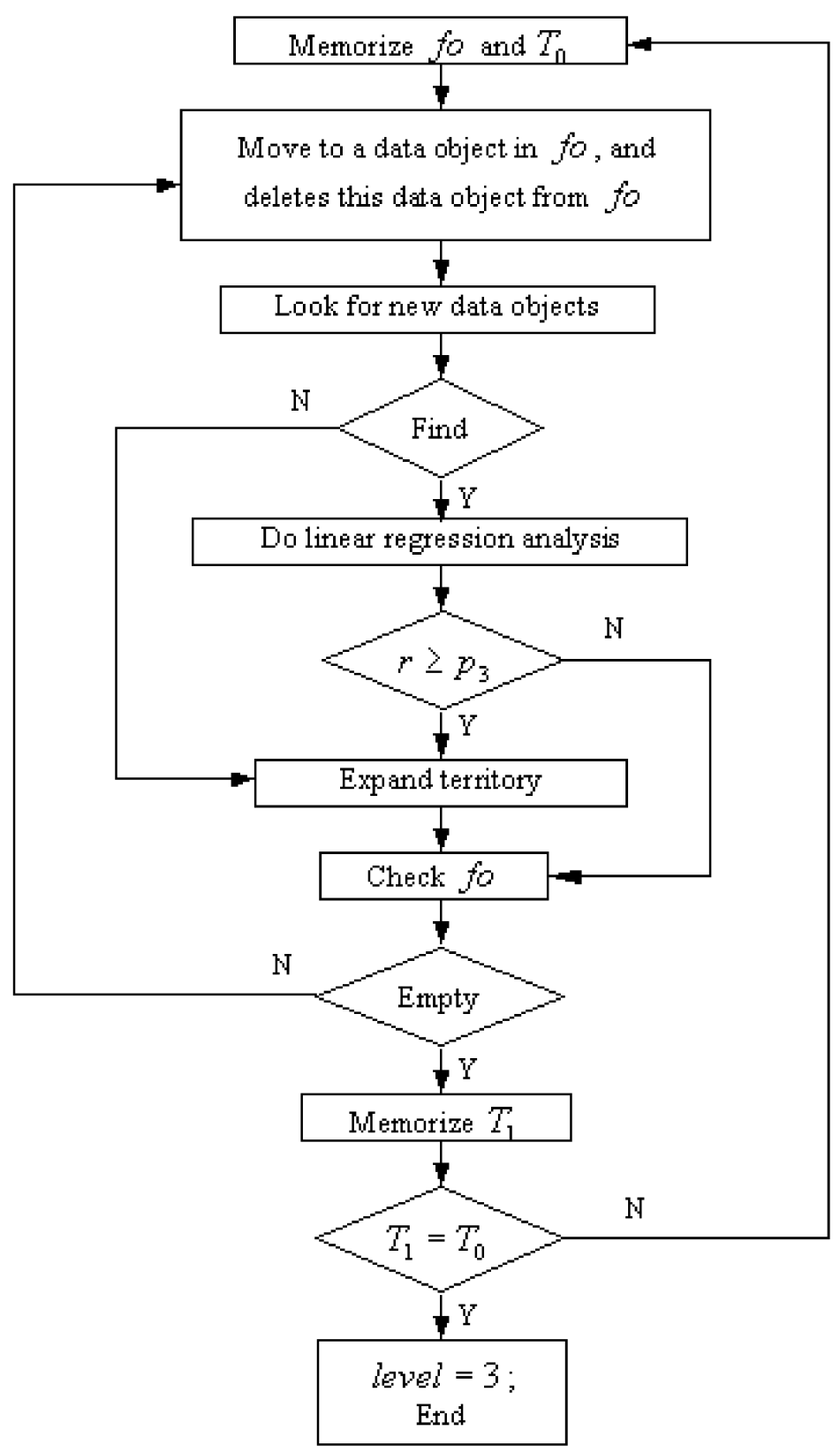

Fig. 7. The process of expanding territory. 


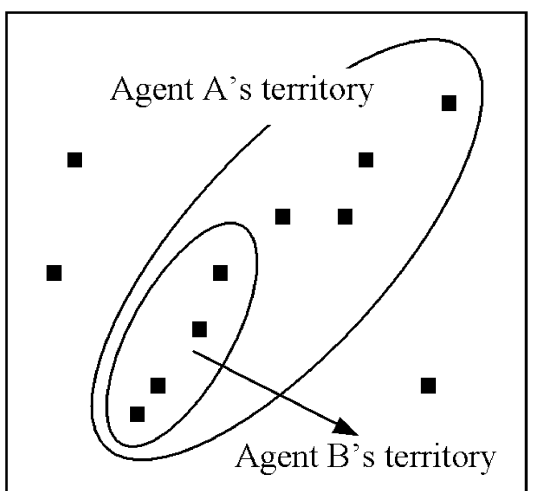

Fig. 8. Agent A eats agent B.

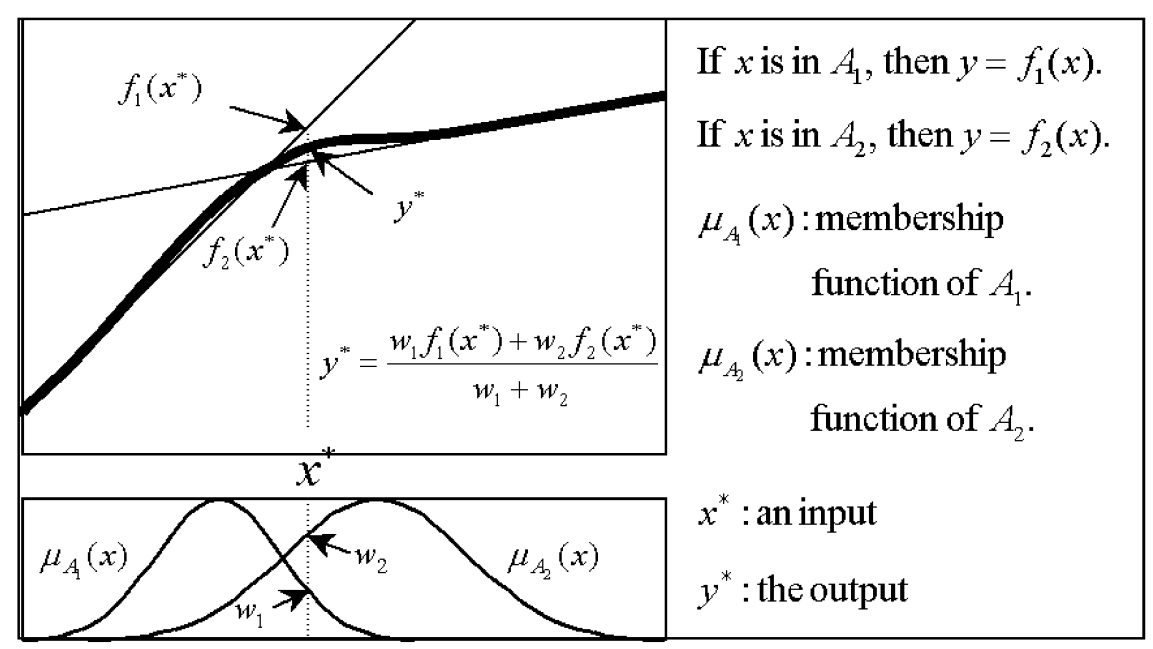

Fig. 9. An example of fuzzy model. 


\section{Forming Global Model}

When there are only level-3 agents left in the data space, they begin to build the global model that is formed by integrating local linear models. Here the integration is done by introducing membership function (Fuzzy model). Fig. 9 shows a simple example with two local linear models or two rules ${ }^{10}$. For the first local linear model (or rule: $x$ is small), the membership function is $\mu_{1}(x)$; for the second local linear model (or rule: $x$ is large), the membership function is $\mu_{2}(x)$. For a certain $x$ value $x=x^{*}$, if $\mu_{1}\left(x^{*}\right)=c, \mu_{2}\left(x^{*}\right)=d$, the output of the first local linear model is $y_{c}$, and the output of the second is $y_{d}$, then the estimation of $y$ is:

$$
y^{*}=\frac{c \cdot y_{c}+d \cdot y_{d}}{c+d} .
$$

If there are $H$ level-3 agents left in the data space, the set of these agents is:

$$
A=\left\{A_{1}, A_{2}, \cdots, A_{H}\right\} .
$$

For every agent $A_{i} \in A(i=1,2, \cdots, H)$, it holds a local linear model $y_{i}=$ $f_{i}(x)=a_{i} x+b_{i}$. Suppose that $w_{i}(x)$ is $x$ 's membership value for $A_{i}$, then the global model is:

$$
y=\frac{\sum_{i=1}^{H} w_{i}(x) f_{i}(x)}{\sum_{i=1}^{H} w_{i}(x)} .
$$

In Eq. (6), we can see every surviving agent contributes to the final global model with different weight. Corresponding to the 'competition' that we have used when describing the process of agents expanding their territories, we'd like to say agents cooperate with each other to build the global model.

Now the problem is how to get $w_{i}(x)(i=1,2, \cdots, H)$. Three factors are considered. The first is agent $A_{i}$ 's strength, the second is the correlation coefficient of the local linear model built by agent $A_{i}$, and the third is the distance between $x$ and agent $A_{i}$.

As discussed in Section 3, an agent's strength indicates its power to build global model. We define an agent's strength in the following way. If there are $N$ data objects in the data space and $M_{i}$ data objects in agent $A_{i}$ 's territory, then $A_{i}$ 's strength is:

$$
s_{i}=\frac{M_{i}}{N} .
$$

To define the distance between $x$ and agent $A_{i}$, we first define agent $A_{i}$ 's center in the $X$-dimension. Suppose for the data object $o_{j}\left(j=1,2, \cdots, M_{i}\right)$, its value in the $X$-dimension is $x_{j}$, then agent $A_{i}$ 's center in the $X$-dimension is:

$$
C_{i x}=\frac{\sum_{j=1}^{M_{i}} x_{j}}{M_{i}} .
$$


The distance between $x$ and agent $A_{i}$ is defined as:

$$
d_{i}(x)=\left|x-C_{i x}\right|
$$

And $w_{i}$ is defined as:

$$
w_{i}(x)=k_{i} \frac{s_{i} r_{i}}{e^{d_{i}(x)}} .
$$

Here $r_{i}$ is the correlation coefficient of the local linear model built by agent $A_{i}$, and $k_{i}$ is a design parameter that will be used to minimize the mean squared residual of the global model. Suppose the set of $k_{i}$ for $A$ in Eq. (5) is:

$$
K=\left\{k_{1}, k_{2}, \cdots, k_{H}\right\}
$$

It is possible that the set $K$ has too many elements (parameters) in it, for example, if there are 1000 agents surviving, then the number of elements (parameters) in set $K$ will be 1000 . It will be difficult to optimize so many parameters with traditional optimization methods. So instead of aiming at optimization, the strategy here is to improve $K$ with certain resources that users can afford. The following genetic algorithm will be used to identify $K$.

- Step 1: Randomly initialize a set that includes $L K$, as shown in Eq. (12):

$$
\mathbf{K S}^{\mathbf{t}}=\left\{K_{1}^{t}, \cdots, K_{L}^{t}\right\}(t=0)
$$

Here the superscript $t$ denotes time step.

- Step 2: For each $K_{j}^{t}(j=1, \cdots, L)$, according to Eq. (6), Eq. (7), Eq. (8), Eq. (9) and Eq. (10), calculate the residual sum of squares $\delta_{j}$ of the global model and get a set:

$$
R^{t}=\left\{\delta_{1}^{t}, \cdots, \delta_{L}^{t}\right\}
$$

Suppose $\delta_{\max }$ is the maximum value in $R^{t}$, then for every $K_{j}^{t}(j=1,2, \cdots, L)$ in $\mathbf{K S}^{\mathbf{t}}$, its possibility of being the genome of the next generation $K$ is:

$$
P\left(K_{j}^{t}\right)=\frac{\delta_{\max }^{t}-\delta_{j}^{t}}{\sum_{l=1}^{L}\left(\delta_{\max }^{t}-\delta_{l}^{t}\right)} .
$$

- Step 3: $t=t+1$, generate the next generation $\mathbf{K S}^{\mathbf{t}}$ by crossover and mutation. If $t<V$, go to step 2; otherwise go to step 4 .

- Step 4: If $\delta_{q}^{t}$ is the minimum value in $R^{t}$, then the $K_{q}^{t}$ in $\mathbf{K S}^{\mathbf{t}}$ is selected to build the global model.

- Step 5: End. 
In the above, for generating enough genomes and better results, we recommend that $L$ be not smaller than 10 and $V$ be not smaller than 500 . Introducing the genetic algorithm here does not mean people have to use it to identify $K$. People can use other optimization methods according to actual situations.

The global model given by Eq. (6) is based on the standardized data set. Corresponding to Eq. (1) and Eq. (2), for getting the global model for the raw data set, linear conversions should be done according to Eq. (15) and Eq. (16) :

$$
\begin{aligned}
x^{\prime} & =\frac{x_{\max }^{\prime}-x_{\min }^{\prime}}{\text { SpaceXSize }} x+x_{\min }^{\prime}, \\
y^{\prime} & =\frac{y_{\max }^{\prime}-y_{\min }^{\prime}}{\text { SpaceYSize }} y+y_{\min }^{\prime} .
\end{aligned}
$$

Now we give a simple example to show how the level-3 agents build the global model. As shown in Fig. 10, there are 10 data objects in the data space. At the beginning, 10 agents are sent into the space. After the competition of survival,there are only two level-3 agents left in the data space: agent $A_{1}$ and agent $A_{2}$. The number beside every data object indicates the data object's value in the $X$-dimension. There are 6 data objects in agent $A_{1}$ 's territory and 5 data objects in agent $A_{2}$ 's territory. According to Eq. (7), The strengths of these two agents are:

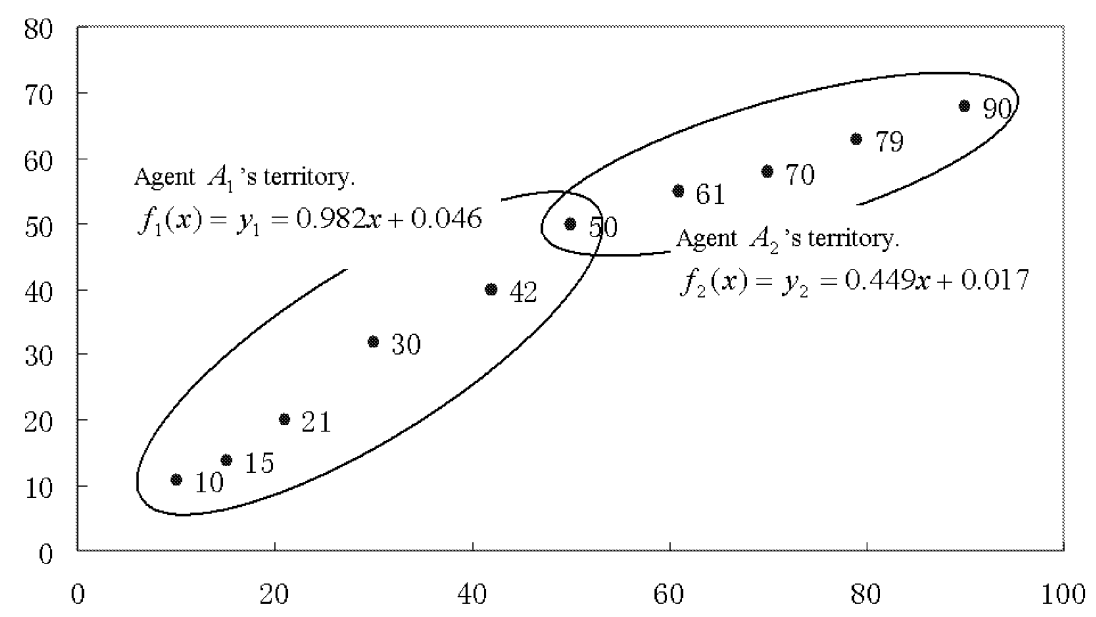

Fig. 10. A simple example for building global model.

$$
\begin{aligned}
& s_{1}=6 / 10=0.6, \\
& s_{2}=5 / 10=0.5 .
\end{aligned}
$$


According to Eq. (8), the centers in the $X$-dimension of these two agents are:

$$
\begin{gathered}
C_{1 x}=(10+15+21+30+42+50) / 6=28, \\
C_{2 x}=(50+61+70+79+90) / 5=70 .
\end{gathered}
$$

Then, according to Eq. (9), the distances between $x$ and these two agents are:

$$
\begin{aligned}
& d_{1}=\left|x-C_{1 x}\right|=|x-28|, \\
& d_{2}=\left|x-C_{2 x}\right|=|x-70| .
\end{aligned}
$$

Suppose $r_{1}=0.9, r_{2}=0.96, k_{1}=0.2$, and $k_{2}=0.3$, according to Eq. (10), we can get:

$$
\begin{aligned}
& w_{1}(x)=k_{1} \frac{s_{1} r_{1}}{e^{d_{1}(x)}}=0.108 e^{-|x-28|} \\
& w_{2}(x)=k_{2} \frac{s_{2} r_{2}}{e^{d_{2}(x)}}=0.144 e^{-|x-70|} .
\end{aligned}
$$

Then, according to Eq. (6), we can get the global model of the data space:

$$
y=\frac{\sum_{i=1}^{2} w_{i}(x) f_{i}(x)}{\sum_{i=1}^{2} w_{i}(x)}=\frac{3 e^{|x-70|}(0.982 x+0.046)+4 e^{|x-28|}(0.449 x+0.017)}{3 e^{|x-70|}+4 e^{|x-28|}}
$$

This example just focuses on how to integrate local linear models to get the global model. It is just an assumption that there are two level-3 agents left in the data space after competition.

In real applications, it is possible there are hundreds of level-3 agents after competition. We can not know the real number of level-3 agents until the multiagent system gives us the result. To a great extent, the number of level- 3 agents depends on how we set the design parameters $p_{1}, p_{2}$ and $p_{3}$. For setting these three design parameters, it is necessary to join logic and intuition together and avoid combinatorial explosion. Generally, the bigger the $p_{1}$ is set, the less agents will survive, because with bigger visions (see Fig. 4), the agents have more chance to eat each other; the bigger the $p_{2}$ is set, the less agents can survive, because with a bigger $p_{2}$ there will be less agents which can evolve into level-2 agents (see Fig. 5); and the bigger the $p_{3}$ is set, the more agents can survive, because with a bigger $p_{3}$ it will be more difficult for agents to expand their territories, and then the agents have smaller chance to eat each other (see Fig. 7). In the next section, we will give two examples that apply the approach presented in this paper and show how informed intuition affect modeling.

\section{Two Numerical Examples}

In this section, we will use the approach presented in this paper to deal with two real two-dimensional numerical cases. 
Table 1. Time of fall in seconds vs. radius of falling sphere of paraffin.

\begin{tabular}{cc|cc}
\hline \multicolumn{2}{c|}{ Raw dataset } & \multicolumn{2}{c}{ Standardized dataset. } \\
\hline$x^{\prime}$ & $y^{\prime}$ & $x$ & $y$ \\
0.00415 & 1.5 & 200 & 0 \\
0.00372 & 3.2 & 176 & 3 \\
0.00369 & 2.5 & 175 & 2 \\
0.00354 & 3.4 & 167 & 3 \\
0.00343 & 3.75 & 161 & 4 \\
$\vdots$ & $\vdots$ & $\vdots$ & $\vdots$ \\
0.000527 & 106 & 1 & 172 \\
0.0005 & 123.2 & 0 & 200 \\
\hline$x_{\text {max }}^{\prime}=0.00415$ & $y_{\max }^{\prime}=123.2$ & & \\
$x_{\text {min }}^{\prime}=0.0005$ & $y_{\text {min }}^{\prime}=1.5$ & & \\
\hline
\end{tabular}

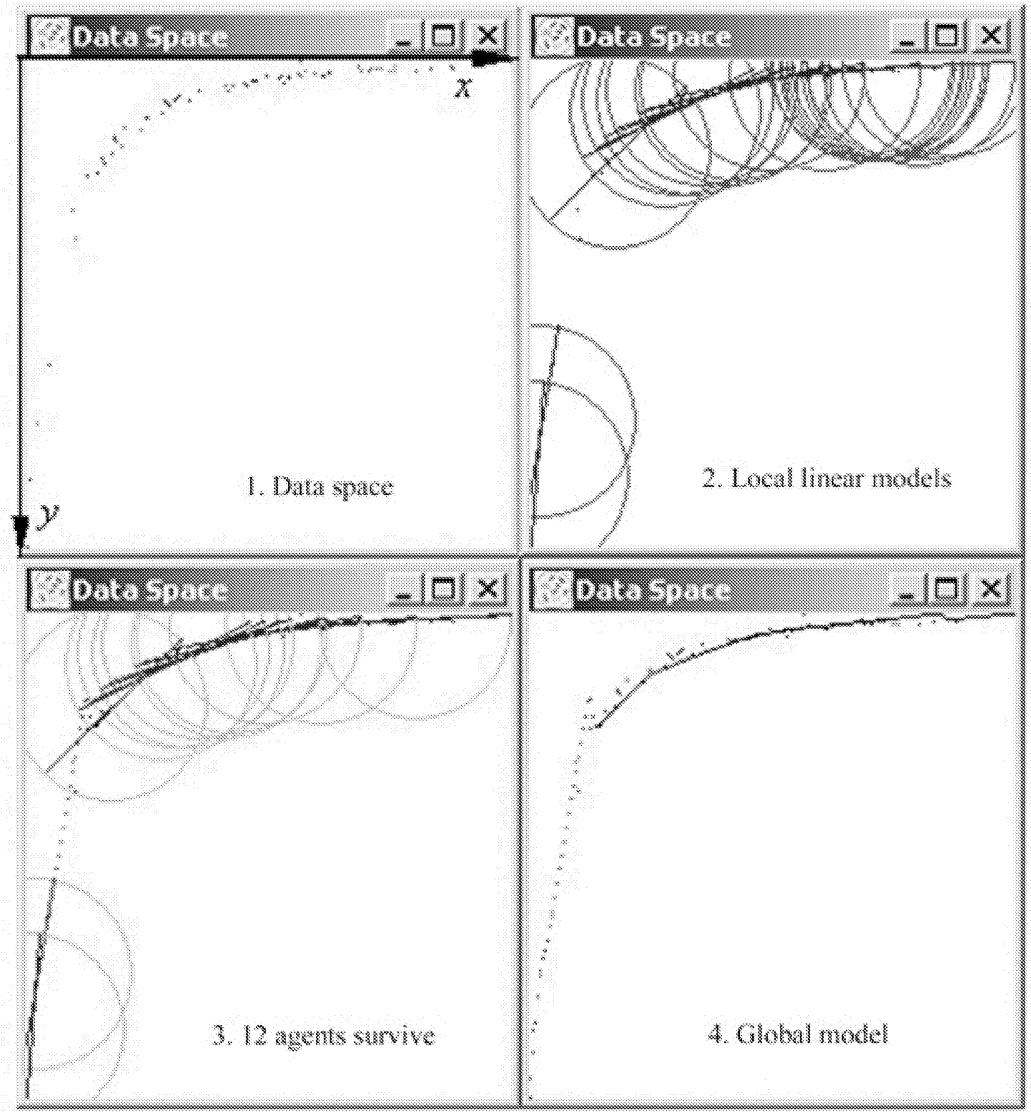

Fig. 11. The first example. 


\subsection{Example 1}

The data set in Table 1 is time of fall in seconds vs. radius of falling sphere of paraffin in cm. It comes from UCI (University of California, Irvine) machine learning repository ${ }^{11}$. We set SpaceXSize $=$ SpaceY Size $=200$. The raw data set is standardized according to Eq. (1) and Eq. (2). If we look every $o_{i}\left(x_{i}, y_{i}\right)$ as a data object, there are 68 data objects in the data space. As shown in Fig. 11_1, a point indicates a data object.

We send 68 agents into this data space and set $p_{1}=40, p_{2}=0.7, p_{3}=0.99$, $L=40$, and $V=1000$. For the genetic algorithm, the crossover rate is set to be 0.7 and the mutation rate is set to be 0.02 for identifying the $K$ in Eq. (11). Fig. 11_2 shows the local linear models built by agents at the beginning. After agents expand their territories and compete to survive, only 12 agents survive. Fig. 11_3 shows the local linear models built by these 12 agents. In Fig. 11_2 and Fig. 11_3, one circle means an agent, but that does not mean an agent is a circle. One circle is just a symbol of an agent. Fig. 11_4 shows the global model built by integrating those local linear models built by surviving agents. Fig. 12 shows the residual sum of squares $\left(\sum_{i=1}^{68} e_{i}^{2}=\sum_{i=1}^{68}\left(y_{i}^{\prime}-\hat{y}_{i}^{\prime}\right)^{2}\right)$ at each step of the genetic algorithm. We can see that although the genetic algorithm can't ensure to find the optimizing solution, it can improve the solution little by little.

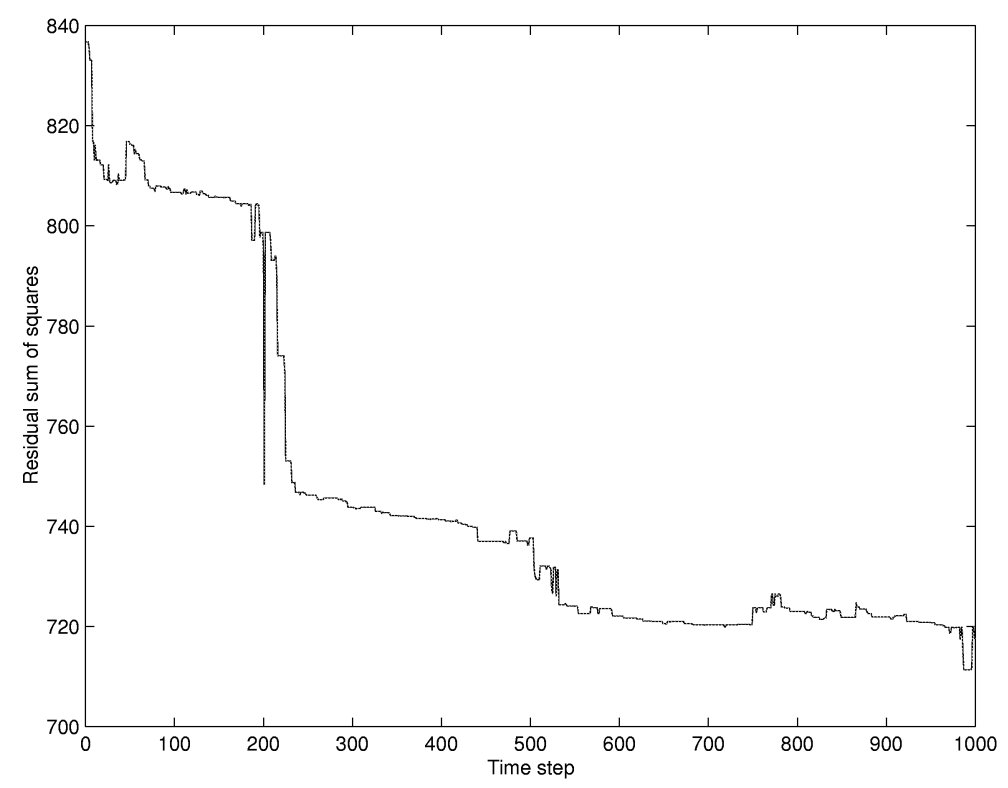

Fig. 12. Residual sum of squares at each step of the genetic algorithm (Example 1).

In a traditional way, people will build a model like $\ln y^{\prime}=a \ln x^{\prime}+b$ for this raw 


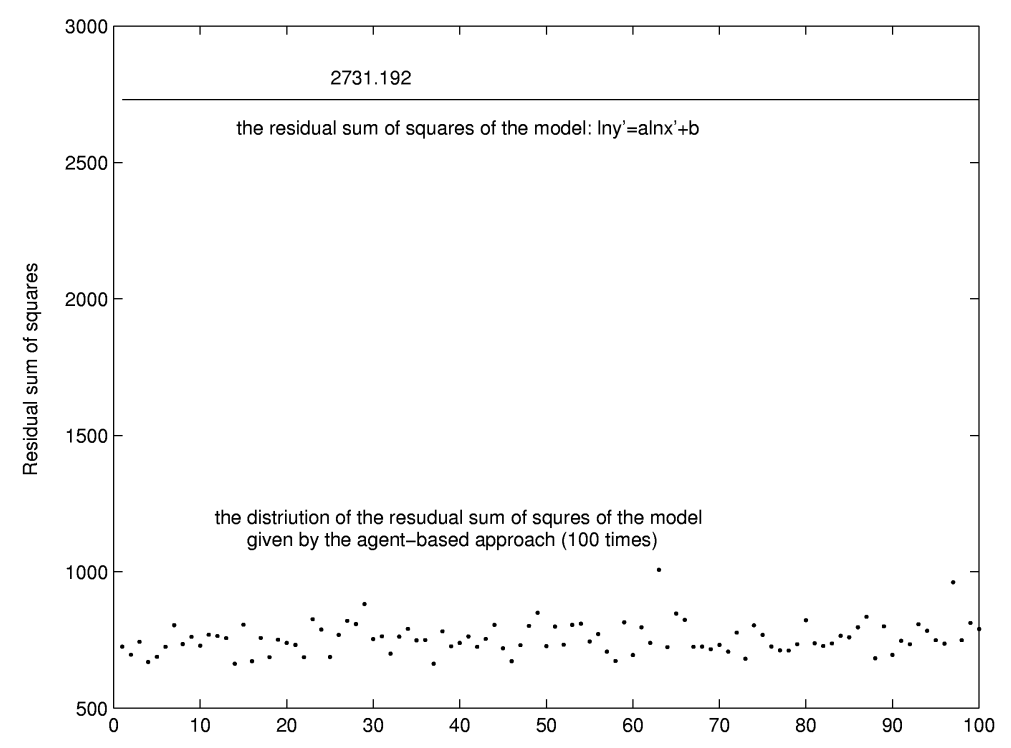

Fig. 13. Distribution of the residual sum of squares (Example 1).

data set. After using regression analysis, the model will be $y^{\prime}=5.012 \times 10^{-5} x^{-1.9}$, and the residual sum of squares will be 2731.192 . The agent-based approach introduced in this paper is a kind of soft computing method. The residual sum of squares is not absolutely the same at every time when we run the programming. We ran the programming of the agent-based approach for 100 times, and after doing linear conversions according to Eq. (15) and Eq. (16), the average value of the residual sum of squares of the model in Fig. 11_4 is 769.116. Fig. 13 shows the distribution of the residual sum of squares of the 100 times. Compared to the model given by the traditional approach, the global model given by the agents fits the data objects better.

\subsection{Example 2}

The data set in Table 2 is the personal income and gross saving of America from 1929 to 2000 in billions of dollars ${ }^{12}$. We also set SpaceX Size $=$ SpaceY Size $=200$. There are totally 72 data objects in the data space. The settings of the parameters $\left(p_{1}, p_{2}, p_{3}, L\right.$ and $\left.V\right)$ are just the same as in the example 1 . And for the genetic algorithm, the crossover rate is set to be 0.7 and the mutation rate is set to be 0.02 . In a traditional way, people will build a model like $y^{\prime}=a x^{\prime}+b$ for this raw data set. After using regression analysis, the model will be $y^{\prime}=0.205 x^{\prime}+38.864$, and the residual sum of squares will be 945182.863 . We also ran the programming of the agent-based approach for 100 times, and after doing linear conversions according 
Table 2. Personal income and gross saving of America.

\begin{tabular}{cc|cc}
\hline \multicolumn{2}{c|}{ Raw dataset } & \multicolumn{2}{c}{ Standardized dataset } \\
\hline$x^{\prime}$ & $y^{\prime}$ & $x$ & $y$ \\
85.3 & 19.2 & 1 & 2 \\
76.5 & 14.9 & 1.0 & 1.0 \\
$\vdots$ & $\vdots$ & $\vdots$ & $\vdots$ \\
8319.20 & 1785.70 & 200 & 200 \\
\hline$x_{\text {max }}^{\prime}=0.00415$ & $y_{\text {max }}^{\prime}=123.2$ & & \\
$x_{\text {min }}^{\prime}=0.0005$ & $y_{\text {min }}^{\prime}=1.5$ & & \\
\hline
\end{tabular}

to Eq. (15) and Eq. (16), the average value of the residual sum of squares of the model in Fig. 14_2 is 12089.936. Fig. 15 shows the residual sum of squares at each step of the genetic algorithm. Fig. 16 shows the distribution of the residual sum of squares of the 100 times.

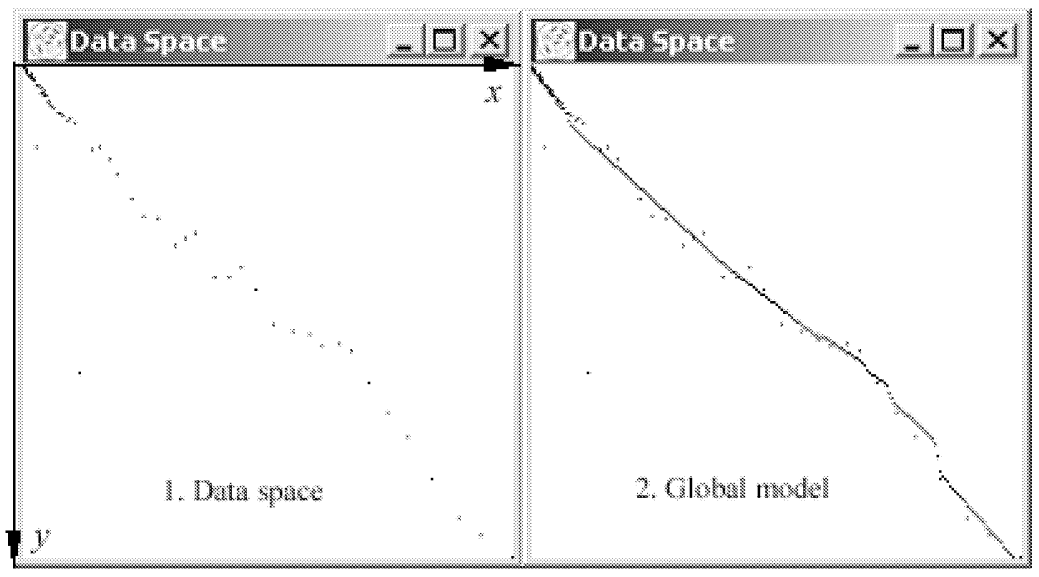

Fig. 14. The second example.

In this example, many people may prefer to select the traditional way to build a simple linear model for the data, because the correlation coefficient $r$ is about 0.971 , very close to 1 . If we set the agent's vision to be large enough (cover all the data space), the model given by the agent-based approach is just the same as the linear model obtained by using regression, as shown in Fig. 17. In this case, just one agent survives. So we can see human's informed intuition can play an important role during the process of modeling.

Neural Networks have been widely used in economic forecasting. Now we compare the performance of the agent-based approach with that of a neural network by using Root-Mean-Square Error $(R M S E)$. In Eq. 17, the $N_{t}$ is the size of the testing set. For example, if $N_{t}=10$, then the final 10 data records (from 1991- 


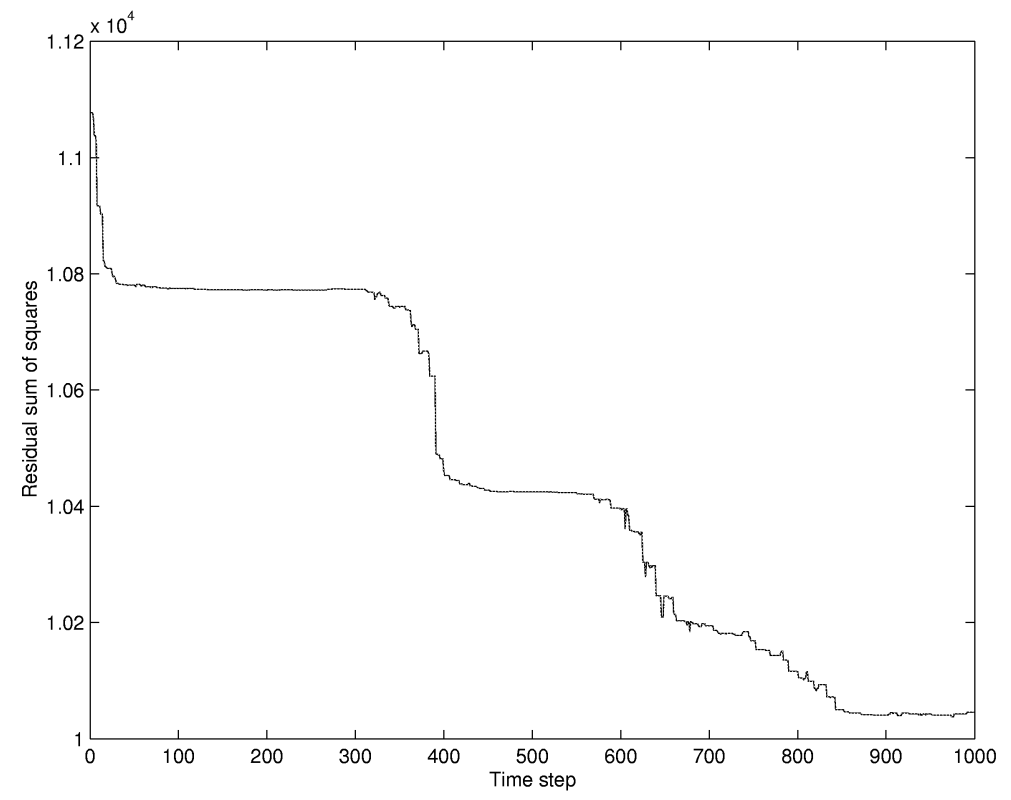

Fig. 15. Residual sum of squares at each step of the genetic algorithm (Example 2).

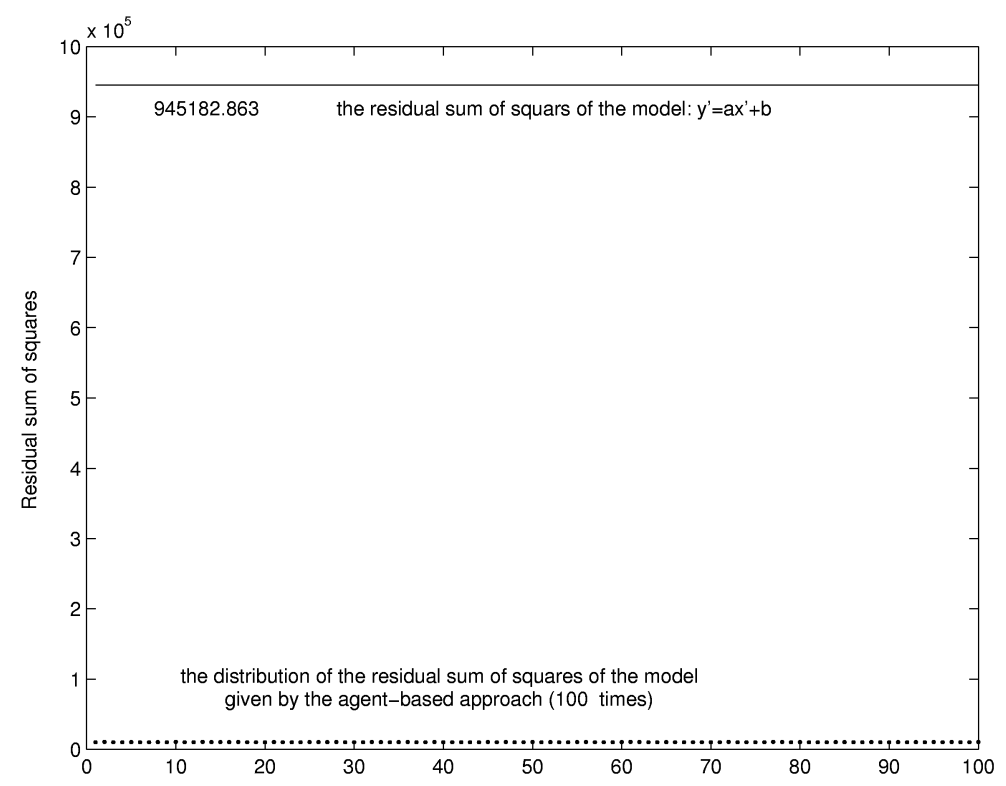

Fig. 16. Distribution of the residual sum of squares (Example 2). 


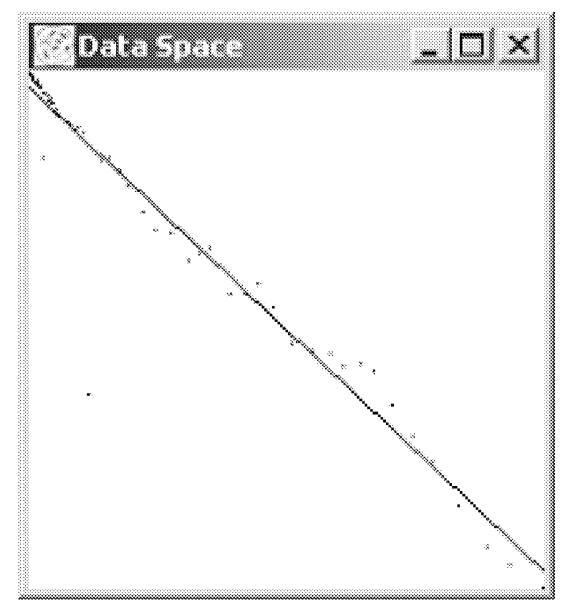

Fig. 17. The same as the model yielded by linear regression.

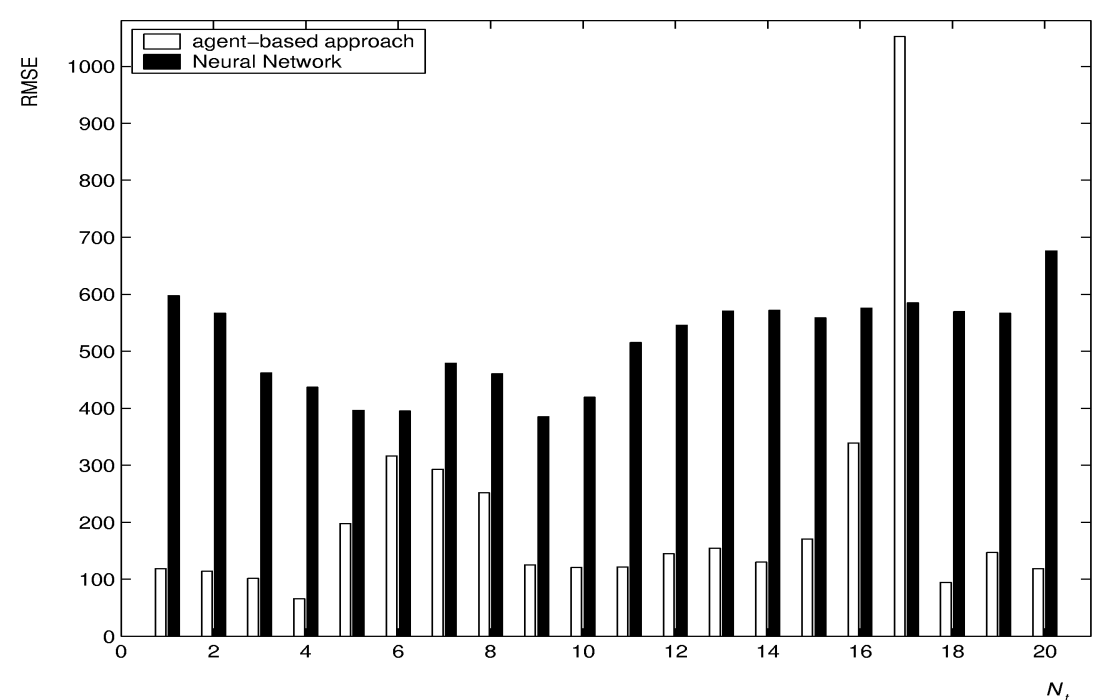

Fig. 18. Comparing the performance of the agent-based approach with that of a neural network. 
2000) are used as testing data, while the 62 data records from 1929 to 1990 are used as training data. The smaller the RMSE, the better the performance of the model. The neural network we have used is a backpropagation network which has been programmed into the software Neunet ${ }^{13}$. With 3 nodes, we trained the neural network for $1 \times 10^{5}$ times in each forecasting.

$$
R M S E=\sqrt{\frac{1}{N_{t}} \sum_{i=1}^{N_{t}}\left({\hat{y^{\prime}}}_{i}-y_{i}^{\prime}\right)^{2} .}
$$

In Fig. 18, the $N_{t}$ is set from 1 to 20 . We can see that the performance of the agent-based approach is always better than that of the neural network except when $N_{t}=17$. Of course, the comparison doesn't prove that the performance of the agent-based approach is better than Neural Networks at any occasion.

\section{Conclusion and Future Work}

This paper presented an agent-based approach to identify prediction models in two-dimensional data spaces. The advantage of this approach is it builds prediction models in a bottom-up way by competition and cooperation among agents; that is to say, it does not need people to give a certain formula in advance (which is very difficult in most of time) because the agents will find the right formula. Although it has not been proven that the models yielded by this approach always are better than those yielded by other approaches, this approach shows and proves the idea that agents can produce the global model by cooperation and competition with some built-in knowledge and simple rules. The models built by this approach are something like emergences. This idea is different from the one that says some software, such as Microsoft Excel, can give models according to input data. In fact, these softwares just do some calculating work, not build models; the models are built by humans. This idea is also a little different from that of the neural network which also does not need people to give a certain formula in advance. The basic elements of neural network are nerve cells which have no intelligence themselves. The agents in this paper are equipped with some knowledge and intelligence.

Now we just use this approach to deal with two-dimensional numerical data. In the real world, most of data are multi-dimensional, both numerical and categorical. For multi-dimensional data, a very difficult problem is to select variables. That is to say, in a subspace, some variables are irrelevant to the problem we are investigating; we would need to identify those variables that are relevant to the problem for getting better prediction result. Y. Nakamori and M. Ryoke have described this problem in detail and have succeed in developing an interactive approach in which human knowledge or intuition can play an important role ${ }^{9}$. For categorical data, the problem is how to define similarity. Based on the basic thinking of this paper, we plan to develop an agent-based approach to deal with multi-dimensional data, both numerical and categorical. 


\section{Acknowledgements}

We appreciate the help of Professor Robert B. DiGiovanni, a Visiting Professor of Technical Communication, Japan Advanced Institute of Science and Technology in reading the manuscript.

\section{References}

1. G. Ballot and E. Taymaz, "Technology change, learning and macro-economic coordination: An evolutionary model", Journal of Artificial Societies and Social Simulation, vol. 2, No. 2, 1999.

2. M. John, "Active Nonlinear Tests (ANTs) of Complex Simulations Models", Management Science, 44(6), June, 1998, pp. 820-830

3. J. Doran, M. Palmer, N. Gilbert, and P. Mellars, "The EOS project: modeling Upper Palaeolithic social change", in Simulating Societies, eds. N. Gilbert and J. Doran (London: UCL Press, 1994) pp.195-222.

4. O. Thomas, "Computer Simulation: The Third Symbol System", Journal of Experimental Social Psychology, 24(5), September, 1998, pp. 381-392.

5. B. Roy, "Using Agents to Make and Manage markets Across a Supply Web: Replacing Central, Global Optimization with a Distributed, Self-Organizing Market Approach", Complexity, 3(4): pp.31-35, 1998.

6. W. Bunn Derek and S. Oliveira Fernando, "Agent-Based Simulation-An Application to the New Electricity Trading Arrangements of England and Wales", IEEE Transition on Evolutionary Computation, vol. 5. No. 5. October 2001.

7. J. Han and K. Micheline,Data Mining - Concepts and Techniques, (Morgan Kaufmann Publishers, 2000).

8. G. Majone, "The craft of applied systems analysis", Rethinking the Process of Operational Research and Systems Analysis, R. Tomlinson and I. Kiss, Eds. Oxford: Pergamon Press, 1984, pp. 143-157.

9. Y. Nakamori and M.Ryoke, "Identification of Fuzzy Prediction Models Through Hyperellipsoidal Clustering", IEEE Transaction on Systems, Man, and Cybernetics, vol. 24, No. 8, August 1994.

10. T. Takagi and M. Sugeno, "Fuzzy identification of Systems and its applications to modeling and control", IEEE Transaction on Systems, Man, and Cybernetics, vol. SMC-15, No. 1, pp.116-132, 1985.

11. UCI machine learning repository, available at: http://www.ics.uci.edu/ mlearn/MLRepository.html.

12. NIPA (National Income and Product Accounts) Data, available at:http://www.bea.doc.gov/bea/dn/nipaweb/Index.asp.

13. Available at: http://www.cormactech.com/neunet/. 